Cahiers québécois de démographie

\title{
Classe, sexe et trajectoire socio-professionnelle
}

\section{Le cas de l'immigration hailtienne au Québec}

\section{Class, Sex and Socio-Professional Trajectory}

The Case of Haitian Immigration in Quebec

\section{Clase, sexo y trayectoria socio-profesional}

\section{El caso de la inmigración haitiana}

\section{Ghyslaine Neill}

Volume 14, numéro 2, octobre 1985

Démographie sociale

URI : https://id.erudit.org/iderudit/600574ar

DOI : https://doi.org/10.7202/600574ar

Aller au sommaire du numéro

Éditeur(s)

Association des démographes du Québec

ISSN

0380-1721 (imprimé)

1705-1495 (numérique)

Découvrir la revue

Citer cet article

Neill, G. (1985). Classe, sexe et trajectoire socio-professionnelle : le cas de l'immigration haïtienne au Québec. Cahiers québécois de démographie, 14(2), 259-273. https://doi.org/10.7202/600574ar
Résumé de l'article

Si plusieurs travaux ont mis en lumière le rôle de l'immigration haïtienne dans la satisfaction des besoins en main-d'oeuvre du Québec, peu de recherches empiriques ont exploré les mécanismes par lesquels les immigrantes et les immigrants sont amenés à jouer ce rôle. C'est le sexe et l'appartenance de classe dans le pays d'origine qui conditionnent largement les trajectoires socio-professionnelles des immigrants haïtiens au Québec. Ces deux facteurs déterminent en effet un processus spécifique d'acquisition des qualifications, à partir desquelles peuvent se constituer des instruments plus ou moins négociables sur le marché du travail au Québec. 
Cahiers québécois de démographie

Vo1. 14, no 2, octobre 1985

\title{
Classe, sexe et trajectoire socio-professionnelle: le cas de l'immigration haïtienne au Québec
}

\author{
Ghyslaine NEILL*
}

\section{INTRODUCTION}

Depuis une quinzaine d'années, l'immigration haïtienne au Québec constitue un phénomène social important. Son évolution, son ampleur et ses caractéristiques particulières ont suscité un grand intérêt. Les diverses questions reliées à l'insertion de la population haïtienne dans les structures de la société québécoise ont d'ailleurs fait 1 'objet de plusieurs recherches ${ }^{1}$.

C'est à partir des résultats du projet "Famille, travail et réseaux migratoires" ${ }^{2}$ que nous voulons dans cet article caractériser $1^{\prime}$ insertion des immigrantes et des immigrants hä̈tiens sur le marché du travail québécois, ainsi que le déroulement de leur trajectoire socio-professionnelle. Conme l'originalité de cette recherche se trouve dans l'importance qu'elle accorde à la vie des immigrants dans le contexte de leur pays d'origine, nous tenterons également de voir dans quelle mesure les modes d'insertion et les diverses trajectoires au Québec sont liées à leur cheminement professionnel en Haiti.

Pour répondre à ces questions, nous avons d'abord étudié le secteur d'activité et le type d'emploi occupés au Québec. Mises en relation, ces informations nous permettent d'observer la rotation des emplois et les passages entre les divers secteurs d'activité, selon le sexe, l'année d'arrivée au Québec et le statut réglementaire à l'arrivée.

* Université de Montréal, Centre de recherches caraïbes.

Une version préliminaire de cet article a été présentée lors du 8 e colloque biennal de la Sociëté canadienne des études ethniques tenu à Montréal du 16 au 19 octobre 1985.

1. Mentionnons à cet égard les travaux de F. Bernèche et de J.-C. Martin sur les regroupements de la population haïtienne à Montréal, ainsi que coux de C. Pierre-Jacques sur l'intégration scolaire des enfants haïtiens, réalisés au Centre de recherches caraïbes de 1'Université de Montréa1.

2. Cette recherche, réalisée au Centre de recherches caraïbes (CRC), fut dirigée par M. Labelle (Université du Québec à Montréa1), S. Larose $(C R C)$ et V. Piché (Université de Montréal). Ce projet fut en majeure partie financé par le Fonds pour la Formation de Chercheurs et l'Aide à la Recherche (FCAR) du Québec et le Conseil de Recherches en Sciences Humaines du Canada (CRSHC). 
Puisque les diverses destinations professionnelles prises par les immigrants haïtiens au Québec nous renvoient nécessairement à leur qualification, nous allons ensuite en étudier le processus d'acquisition dans leur pays d'origine. En effet, la formation et l'expérience de travail acquises en Haĭti constituent pour les individus un ensemble d'instruments plus ou moins négociables sur le marché du travail québécois, selon la conjoncture. Ce processus d'acquisition des qualifications sera donc mis en relation avec deux variables-clés, l'appartenance de classe en Haïti et le sexe, qui en déterminent largement les modalités.

Mais avant d'aborder ces questions, il nous semble important de rappeler les principales caractéristiques de l'immigration haïtienne au Québec, tant dans ses aspects statistiques que contextuels, cela à partir des travaux les plus récents.

\section{L'IMMIGRATION HAITIENNE AU QUÉBEC : ASPECTS STATISTIQUES ET CONTEXTUELS}

Les diverses études concernant les aspects statistiques de l'immigration haítienne au Québec ont permis de faire d'abord ressortir le caractère hétérogène de sa main-d'oeuvre (Dejean, 1978; Tardieu, 1979; Nei11, 1983; Normandeau et Piché, 1983). En effet, l'analyse des flux migratoires annuels, basée sur les statistiques du Ministère de l'immigration du Québec, nous révèle en fait 1 'existence de deux courants migratoires distincts, ayant évolué sur deux périodes. La première période, de 1968 à 1972, est caractérisée par une augmentation des flux annuels, une main-d'oeuvre jeune et qualifiée se dirigeant principalement vers des emplois de type professionnel et technique, et une forte proportion d'individus indépendants. Entre 1973 et 1980 ces tendances s'inversent. Ainsi, la proportion d'individus indépendants est de plus en plus faible et la main-d'oeuvre, de moins en moins qualifiée, se dirige vers le secteur manufacturier et celui des services.

Pour comprendre le caractère hétérogène de la main-d'oeuvre haïtienne et son rôle dans la satisfaction des besoins en main-d'oeuvre du Québec, il est nécessaire de cerner le contexte de son émigration ainsi que celui de son immigration, puisqu'il "fournit une perspective historique et structurelle à la présence différenciée d'un groupe quel qu'il soit et à son rôle dans une société d'accueil" (Labelle, Larose et Piché, 1983 : 87).

C'est dans le contexte d'une économie dépendante qu'il faut d'abord situer l'émigration haïtienne des années 1960 vers les pays industrialisés ${ }^{3}$. En effet, le développement des activités en fonction de l'extérieur (sources premières de devises étrangères pour l'achat de biens importés et destinés principalement à la classe dominante), le contrôle étranger sur le commerce

3. Pour une analyse des mouvements migratoires internes (de la campagne vers les villes), voir notamment Baril, Gregory et Jacques (1985). En ce qui concerne la migration haltienne vers les autres îles des Caraïbes, on peut consulter Holly, Labelle et Larose (1979). 
d'exportation et 1 a production industrielle, la faible production des biens d'équipement et de consommation, le rapatriement des profits à l'étranger et une balance des paiements chroniquement déficitaire, sont à la base des rapports de dépendance qui caractérisent les relations entre les pays industrialisés et Haïti.

Au niveau politique, le régime duvaliériste a contribué non seulement "à exacerber la dépendance structurelle», mais aussi à perpétuer "l'autoritarisme qui a marqué la politique depuis la formation de la République en 1804" (Labelle, Larose et Piché, 1983 : 78).

Si, depuis les années 1960, le mouvement d'émigration vers les pays industrialisés est fortement tributaire des conditions économiques et politiques d'Haïti, le Québec en a pour sa part largement profité. L'immigration haïtienne, principalement à cause de son caractère hétérogène, a en effet joué un rôle important dans la satisfaction des besoins en main-d'oeuvre au Québec.

En observant l'évolution économique du Québec, on assiste, à partir des années 1960, à une augmentation considérable des dépenses publiques, orientées surtout vers les secteurs de la santé, du bien-être et de I'éducation. De 1960 à 1965, les effectifs de l'administration publique augmentent de $53 \%$.

Le développement rapide de 1'appareil étatique québécois a bénéficié de cette main-d'oeuvre jeune et qualifiée que constituait la majeure partie de l'immigration haïtienne à cette époque. De 1968 à 1972, 1'étude des emplois projetés, provenant des statistiques annuelles du Ministère de 1'immigration du Québec, nous montre qu'en effet $35 \%$ (490) des femmes et $55 \%$ (861) des hommes comptaient travailler dans le secteur professionnel et technique, plus précisément dans les domaines de la santé et de l'éducation.

$\mathrm{Au}$ cours des années 1970, le type de main-d'oeuvre fourni par l'immigration hä̈tienne au Québec se modifie sensiblement. Alors que $20 \%$ (280) des Haïtiennes et $16 \%$ (250) des Haïtiens arrivés entre 1968 et 1972 projetaient de travailler dans le secteur de la fabrication et de la réparation, ces proportions passent respectivement à $39 \%$ ( 1 865) pour les femmes et à $49 \%$ (2 926) pour les hommes entre 1973 et 1980 .

Le nouveau profil de la main-d'oeuvre haitienne correspond surtout aux besoins de l'industrie manufacturière québécoise. Malgré une faible augmentation du nombre d'emplois du secteur secondaire entre 1966 et 1976 $(7 \%)$, plusieurs industries de ce secteur nécessitaient une main-d'oeuvre abondante, peu qualifiée et peu rémunérée. Mentionnons à cet égard combien les activités de fabrication au Québec, surtout orientées vers les produits non durables (textile, vêtement, bonneterie, chaussure, etc.), sont reconnues pour leur vulnérabilité, due notamment à l'utilisation d'une technologie archaïque et à une forte concurrence des pays en vole de développement. 
L'analyse des données du recensement de 1971 confirme ces observations : "En combinant les diverses caractéristiques tirées du recensement de 1971, un profil commence à se dessiner quant aux composantes de l'immigration caraïbéenne : ghettos d'emplois pour les femmes et forts taux d'activité pour celles âgées de 25 à 44 ans, transformation de 1'activité économique pour la population nouvellement immigrée qui se concentre davantage dans l'industrie manufacturière" (Bernèche, 1983 : 112).

L'analyse de l'immigration haïtienne au Québec illustre donc bien le processus complexe par lequel il y a transfert de main-d'oeuvre d'une société dépendante à une société industrialisée (Corten, 1972; Holly, Labelle et Larose, 1979), puisqu'elle met en évidence "... les liens organiques qu'entretiennent les pays capitalistes avancés avec les pays de la périphérie. D'un côté, le sous-développement caractérisé par la dépendance face aux capitaux étrangers (surtout américains, mais aussi canadiens et québécois), participant ainsi aux forces génératrices d'émigration; de l'autre, le recrutement sélectif parmi la force de travail ainsi mise en disponibilité" (Labelle, Larose et Piché, 1983 : 73).

\section{INSERTION ET TRAJECTOIRE SOCIO-PROFESSIONNELIF DE LA MAIN-D'OEUVRE HAITIENNE AU QUEEBEC.}

Les analyses statistiques et contextuelles auxquelles nous venons de faire référence permettent d'appréhender à un niveau général le caractère hétérogène de l'immigration haïtienne au Québec, en la resituant dans le cadre d'un processus complexe de transfert de main-d'oeuvre d'une société dépendante vers une société industrialisée. Mais que signifie concrètement ce processus de transfert pour les individus qui l'alimentent ? Quels sont les mécanismes qui jouent et permettent ce transfert ?

Les sources statistiques habituelles ne peuvent être utilisées pour répondre à ce type de questions ${ }^{4}$. En effet, ces sources ne fournissent aucune information sur les milieux de vie dans le pays d'origine. Tout au plus nous amènent-elles à dresser un profil général à partir des niveaux de scolarité et de l'emploi projeté ou occupé à un moment donné.

Les données recueillies par le projet "Famille, travail et réseaux migratoires" rendent possible ce genre d'analyse. En effet, l'utilisation des récits de vie nous a permis non seulement de recomposer la totalité des trajectoires socio-professionnelles des individus au Québec, mais aussi de reconstituer l'ensemble de leur cheminement à partir d'Haïti. De plus, les divers cheminements professionnels des individus ont pu être replacés dans le contexte de leur appartenance de classe tant au Québec qu'en Haïti. Ainsi pouvons-nous mieux caractériser les mécanismes et les déterminants de leur insertion et de leur trajectoire d'emploi au Québec.

4. Pour une critique plus détaillée des statistiques de frontière, voir Tardieu (1979), Neill (1983) et Normandeau et Piché (1983). En ce qui concerne la critique des données de recensement, voir Tardieu (1979). 
En raison même des objectifs du projet ${ }^{5}$, seulement un petit nombre de personnes ont été interviewées. L'échantillon, constitué par la méthode dite "boule de neige" ${ }^{6}$, ne peut prétendre à une représentativité statistique. Néanmoins, les récits de vie font ressortir la diversité des expériences vécues. C'est dans ce sens qu'il demeure important d'analyser ce matériel, puisqu'il permet la formulation d'hypothèses nouvelles pour 1 'étude des mouvements migratoires.

Dans cette enquête, les individus furent sélectionnés sur la base de deux critères : leur appartenance de classe au Québec et leur sexe. C'est l'emploi au Québec qui fut utilisé comme principal indicateur de l'appartenance de classe. Les principaux indices retenus furent donc "l'occupation, la fonction d'exécution ou d'encadrement, le caractère intellectuel ou manuel du travail accompli et la propriété des moyens de production" (Larose, 1985 : 9). Mentionnons dès à présent le cas des propriétaires-chauffeurs de taxi et celui des petits entrepreneurs ethniques, qui ont été classés comme travailleurs autonomes, intégrés au prolétariat québécois. Ces travailleurs autonomes se distinguent des autres, intégrés à la petite bourgeoisie québécoise, puisque "repoussés par le marché du travail, ils sont forcés de créer une petite entreprise vivotante qui leur permet tant bien que mal de subsisterl (Ibid.).

5. Les principaux objectifs étant de définir les modes d'incorporation des familles immigrantes au marché québécois du travail et de caractériser les réseaux migratoires, le projet a été réalisé en deux volets. Le premier, le volet socio-démographique, avait comme objectif d'étudier l'ensemble de la population haïtienne au Québec. Plus précisément, une analyse des flux migratoires annuels et des emplois au Québec a été effectuée à l'aide des statistiques annuelles du Ministère de l'immigration du Québec et du recensement de 1971. C'est à l'intérieur $\mathrm{du}$ volet socio-anthropologique, largement inspiré d'une recherche effectuée à l'Université du Québec à Montréal, sous la direction de M. Labelle, que $1^{\prime}$ on a mené 76 entrevues en profondeur auprès d'un nombre égal de femmes et d'hommes. Ces entrevues semi-dirigées comprenaient quatre grands blocs de questions : le premier concernait les principales caractéristiques démographiques des individus (âge, sexe, statut matrimonial, statut réglementaire, etc.); le second, leur vie en Haĩti (famille, éducation, emploi, etc.); le troisième, leur migration et leurs motifs de départ; et le dernier, leur vie au Québec (emploi, famille, réseaux, rapports hommes-femmes, logement, etc.).

6. Basé sur la méthode "boule de neige", l'échantillon a tout de même été constitué à partir de 26 "sources" différentes. Un maximum de trois personnes par "source" ont donc été sélectionnées pour l'enquête. De plus, notons que cette méthode nous a permis de stratifier l'échantillon selon le sexe des individus et leur emploi occupé au Québec. Les emplois et les secteurs d'activité retenus l'ont d'ailleurs été en fonction des concentrations observées à partir des statistiques du Ministère de 1'immigration du Québec et du recensement de 1971. 
En fait, cela constitue plus "un indice de marginalisation sur le marché du travail que celui d'une appartenance de classe capitalistel (Larose, 1985 : 9). Enfin, d'autres indices comme l'éducation et le revenu furent retenus. Les membres de l'échantillon appartiennent donc à deux groupes principaux : le prolétariat et la petite bourgeoisie.

Compte tenu des choix méthodologiques de l'enquête, nous allons d'abord caractériser l'insertion sur le marché du travail québécois et 1a trajectoire socio-professionnelle des immigrants haïtiens selon leur appartenance de classe au Québec. Ainsi, nous serons en mesure de voir si le type d'insertion et de trajectoire diffère selon les emplois et les secteurs d'activité vers lesquels les immigrants haïtiens se destinent.

Pour chaque groupe, nous allons dans un deuxième temps retracer les divers cheminements professionnels des individus selon leur appartenance de classe en Haiti ${ }^{7}$. De cette façon, nous pourrons voir d'une part dans quelle mesure 1'appartenance de classe en Haïti détermine des cheminements-types en termes de formation et d'expérience de travail acquises, et d'autre part si ces cheminements-types en Haiti ont une incidence sur l'insertion et la trajectoire d'emploi au Québec.

Par souci de brièveté, nous nous limitons ici à un résumé des principaux résultats obtenus. Une analyse plus détaillée, ainsi qu'une présentation plus complète des résultats quantitatifs, sont présentées dans Kempeneers et Neill (1985).

\subsection{Les immigrants haïtiens intégrés au prolétariat québécois}

a) Insertion sur le marché du travail québécois et trajectoire socio-professionnelle

Immigrées au Québec au cours des années 1970, les personnes de l'échantillon intégrées au prolétariat québécois se sont surtout insérées dans deux secteurs d'activité, les services et la manufacture.

Les femmes entrées au Québec comme touristes ont obtenu leur premier emploi surtout dans le secteur des services, comme domestiques ou gardiennes d'enfants, alors que les autres possédant un visa d'immigrante reçue ont d'abord travaillé comme ouvrières en manufacture.

7. L'appartenance de classe des individus en Haliti a été déterminée par rapport à la place qu'lls occupaient dans la structure de production haîtienne. Pour les personnes n'ayant jamais occupé d'emploi "rémunéré" en Halti, on a attribué l'appartenance de classe des parents. Pour une explication plus détaillée, voir Larose (1985). 
$\mathrm{Au}$ contraire des femmes, on ne voit pas pour les hommes de lien entre secteur d'activité du premier emploi et statut réglementaire à l'arrivée au Québec. En effet, les quelques hommes entrés comme touristes ont, comme la plupart des autres, obtenu leur premier emploi dans le secteur manufacturier. Soulignons tout de même que c'est dans les manufactures de textile et de meubles que ces derniers ont obtenu leur premier emploi.

La poursuite de leur trajectoire socio-professionnelle au Québec se fera aussi dans le secteur manufacturier et dans celui des services. L'examen des séquences d'emplois nous montre d'abord que les femmes effectuent de fréquents passages entre le secteur des services et le secteur manufacturier. En fait, c'est souvent après avoir travaillé comme domestiques que les femmes vont par la suite obtenir un emploi dans les manufactures. Ainsi, l'entrée en manufacture correspond fréquemment à la régularisation de leur situation d'immigrante. Si quelques-unes retournent au secteur des services, c'est pour occuper des emplois reliés surtout à 1 'hôtellerie et à l'entretien ménager.

Les trajectoires socio-professionnelles des femmes sont aussi caractérisées par une rotation d'emploi assez élevée. Elles occupent d'abord des emplois de courte durée, allant de quelques semaines à quelques mois, suivis d'emplois de plus longue durée, pouvant aller jusqu'à quelques années. Mais cette "stabilisation" de l'emploi est fort relative, dans la mesure où les mises à pied, les conditions de travail pénibles, les conflits avec les patrons, le harcèlement sexuel, etc., obligent ces femmes à changer d'emploi malgré l'ancienneté procurée par quelques années de service (voir les témoignages recueillis dans Kempeneers et Neil1, $1985: 17-21)$.

Les trajectoires socio-professionnelles des hommes sont également caractérisées par de fréquents passages d'un secteur d'activité à un autre et par une forte rotation d'emploi. Mais si le passage entre secteurs d'activité se fait surtout des services à la manufacture pour les femmes, on remarque l'inverse chez les hommes. En effet, les hommes passent plutôt d'un emploi en manufacture à un emploi dans les services. Par ailleurs, les types d'emploi qu'occupent les hommes sont très différents de ceux qu'occupent les femmes, dans la mesure où ils ont souvent des postes plus spécialisés, autant dans le secteur manufacturier que dans celui des services. Ainsi, les emplois occupés par les hommes dans le secteur des services ne concernent en rien le domaine de la domesticité, puisque ce secteur regroupe des activités relatives au taxi, à la mécanique automobile et aux autres moyens de transport, ainsi qu'à la restauration et 1 'hôtellerie.

Mais d'où proviennent ces immigrantes et ces immigrants haïtiens ? C'est par l'examen de leur cheminement professionnel en Haïti que nous allons répondre à cette question. 
b) Cheminement professionnel en Haïti

Le cheminement professionnel en Haïti des individus intégrés au prolétariat québécois se caractérise d'abord par des expériences fort diverses. Cette diversité contraste d'ailleurs avec l'homogénéité de leur trajectoire au Québec. Elle s'exprime aussi dans l'appartenance de classe en Haiti, puisque la majorité de ces personnes ne proviennent pas seulement du prolétariat haĩtien mais aussi des couches précaires de la petite bourgeoisie.

Les femmes provenant du prolétariat haïtien ont eu en Haïti des activités liées surtout au secteur informel de l'économie. Leur expérience de travail a été acquise dans les "petites activités marchandes" (surtout la vente itinérante et celle faite sur le "pas de la porte") relatives à la commercialisation des biens de consommation (denrées alimentaires de base, tissus, vêtements, etc.), la couture à domicile, la domesticité et la garde d'enfants. Il n'est d'ailleurs pas rare de les voir cumuler plusieurs activités afin d'assurer la survie du ménage.

En termes de formation, ces femmes sont généralement peu sinon pas scolarisées. Quoique la plupart d'entre elles aient terminé l'école primaire, et que certaines aient poursuivi quelque temps leurs études à l'école secondaire, quelques-unes n'ont jamais fréquenté l'école. Le savoir-faire de l'ensemble de ces femmes a, la plupart du temps, été acquis soit par des cours privés (couture, dactylo), souvent non complétés faute de moyens, soit par 1'apprentissage du métier "sur le tas".

A l'opposé des femmes, les hommes provenant du prolétariat haïtien ont acquis leur expérience de travail surtout dans le secteur formel de l'économie haïtienne. De plus, les types d'emplois occupés présentent un éventail beaucoup plus large. Ainsi, la plupart d'entre eux ont été soit travailleurs manuels des entreprises étatiques (garage, transport, cimenterie, etc.) ou ouvriers spécialisés des petites entreprises précaires du secteur privé (cordonnerie, garage, menuiserie, etc.).

La formation scolaire de ces hommes est supérieure à celle des femmes provenant de la même classe. Ayant tous complété leurs études primaires, plusieurs iront poursuivre leurs études à l'école secondaire. Mais, comme les femmes, aucun d'entre eux n'en sortira avec un diplôme. La précarité de leur situation matérielle les poussera plutôt à acquérir un métier par le "système des apprentis".

L'autre groupe important, intégré au prolétariat québécois et fortement représenté dans l'échantillon, est constitué de personnes provenant de ce $\mathrm{qu}^{\prime}$ il est convenu d'appeler les "couches précaires" de la petite bourgeoisie haïtienne. Avant d'examiner les divers cheminements de ces personnes, nous allons d'abord décrire brièvement l'ensemble de leur situation en Haĩti. 
L'expression "couches précaires" signifie en fait la dégradation des conditions d'existence d'une partie de la petite bourgeoisie. "Ces couches comprennent des artisans et gens de métier à leur compte tels ébénistes, cordonniers, tailleurs et couturières, maîtres maçons. Traditionnellement concentrées dans les villes, ces personnes jouissaient anciennement d'un statut qui les rattachait carrément à la petite bourgeoisie tant par leur niveau d'éducation que par leur revenu relativement élevé. La situation sociale de ces petits patrons n'a cessé de se détériorer face à la concurrence des importations. On retrouve également dans cette catégorie, les fils et les filles des couches aisées de la paysannerie pour qui l'éducation a constitué tant une stratégie de promotion sociale qu'une réponse à la dégradation de leurs conditions de vie. Le plus souvent bloquées au baccalauréat, ces personnes occupent des emplois subalternes dans la fonction publique haïtienne... On retrouve également dans ces couches des petits instituteurs ruraux dont le salaire en 1974 n'était que de 40 \$ par mois, des petits boutiquiers" (Larose, 1985 : 15-16).

Les femmes de 1'échantillon issues des couches précaires de la petite bourgeoisie en Haïti, ont eu des emplois reliés surtout au secteur formel de l'économie haïtienne. Leur expérience de travail s'est acquise entre autres dans les secteurs de l'enseignement primaire, des bureaux et du commerce. Malgré cela, la plupart des emplois que ces femmes occupaient étaient plutôt de type occasionnel et surtout très peu rémunérés.

La formation de ces femmes est marquée par un niveau de scolarité supérieur à celui des femmes provenant du prolétariat haïtien et par l'obtention de diplôme (enseignement, secrétariat, etc.).

Comme pour les femmes, les hommes issus des couches précaires de $1 a$ petite bourgeoisie hä̈tienne ont acquis leur expérience de travail dans des emplois reliés au secteur formel de l'économie en Haïti. La plupart ont travaillé dans la fonction publique (petits fonctionnaires et enseignants plutôt mal rémunérés), alors que d'autres ont eu des emplois dans le secteur administratif et commercial du secteur privé (emplois de bureau).

En termes de formation, ils sont en général plus scolarisés que les femmes provenant de la même classe en Haïti, mais possèdent au total moins de diplômes. En fait, ces hommes et ces femmes ne visent pas les mêmes diplômes. Si les femmes se spécialisent dans les domaines de 1 'enseignement du primaire, du secrétariat, des soins esthétiques, etc., les hommes visent des diplômes plus prestigieux, nécessitant des études universitaires en droit, en administration et commerce, en santé, etc. Comme ces diplômes exigent une plus longue scolarité, il arrive souvent que les hommes doivent interrompre leurs études, la famille ne pouvant plus en assumer le coût. C'est ainsi qu'ils se retrouvent petits fonctionnaires de l'État, comme déjà mentionné. 
Il va sans dire que pour les individus intégrés au prolétariat québécois, l'immigration a des implications diverses suivant leur appartenance de classe en Haiti. Ainsi, pour les personnes issues des couches précaires de la petite bourgeoisie haïtienne, 1'immigration au Québec est "vécue comme une véritable chute sociale" (Larose, 1985 : 16). En effet, malgré un niveau de scolarité supérieur à celui des individus issus du prolétariat haïtien, ils occupent les mêmes types d'emploi au Québec. La non-reconnaissance de leurs qualifications et les obligations face à la famille restée en Haïti les amènent donc à prendre le premier emploi qui se présente.

Pour les individus issus du prolétariat haïtien, l'immigration au Québec a pour effet de les confronter à un système de travail fort différent de celui connu en Haïti, un système "dominé par le taylorisme et le fordisme" (Labelle et alii, 1984 : 12). Par exemple, le "savoir-faire" des femmes, acquis tant bien que mal compte tenu de leurs conditions d'existence en Haïti, n'est pas nécessairement "négociable" sur le marché du travail québécois. Quoi qu'il en soit, ce savoir-faire est tout de même largement utilisé, puisqu'on retrouve notamment ces femmes dans les manufactures de textile et dans le travail domestique.

\subsection{Les immigrants hä̈tiens intégrés à la petite bourgeoisie québécoise}

a) Insertion sur le marché du travail québécois et trajectoire socio-professionnelle

L'insertion sur le marché du travail au Québec et la trajectoire socio-professionnelle des personnes intégrées à la petite bourgeoisie québécoise présentent des caractéristiques fort différentes de celles observées pour les personnes insérées dans le prolétariat québécois.

C'est d'abord dans 1a foulée de leur formation que ces femmes et ces hommes ont immigré au Québec pendant les années 1960. Quoiqu'ils ne soient pas tous arrivés comme étudiants, la majeure partie de ces individus ont poursuivi leur formation académique au Québec avant d'entrer sur le marché du travail.

Pour les femmes comme pour les hommes, le premier emploi obtenu relève principalement des secteurs éducatifs et médicaux, ainsi que de celui des affaires et de l'administration publique. C'est cependant par rapport au type d'emploi que l'on note une fois de plus des différences importantes selon le sexe. Ainsi, les femmes du secteur de la santé sont surtout infirmières, alors que les hommes sont presque tous médecins; tandis que celles du secteur des affaires et de l'administration sont employées de bureau plutôt que professionnels comme les hommes. 
$\mathrm{Au}$ contraire des personnes insérées au prolétariat, celles intégrées à la petite bourgeoisie québécoise ont des trajectoires socio-professionnelles beaucoup plus stables. On remarque en effet que malgré une durée de séjour plus longue, la rotation d'emplois est moins importante. De plus, les changements d'emploi ne relèvent pas des conditions de travail, mais plutôt du fait de vouloir accéder à un emploi plus intéressant, sinon plus rémunérateur.

On note aussi que le déroulement de leur trajectoire poursuit la même tendance que leur insertion sur le marché du travail québécois, c'est-à-dire en continuité par rapport à leur formation initiale. En effet, malgré certaines difficultés liées à la reconnaissance de leurs diplômes, ces personnes auront la plupart du temps des emplois correspondant à leur formation.

Enfin, I'examen de ces trajectoires par sexe nous montre que celles des femmes sont beaucoup plus linéaires puisque les changements d'emploi s'effectuent, dans la grande majorité des cas, à 1'intérieur du même secteur d'activité. En ce qui concerne les hommes, les passages entre secteurs d'activité s'accompagnent tout de même d'une continuité en termes de profession exercée.

\section{b) Cheminement professionne1 en Haïti}

Si on examine les cheminements professionnels en Haïti des individus intégrés à la petite bourgeoisie québécoise, on constate une plus grande uniformité qu'en ce qui concerne ceux intégrés au prolétariat. Mais avant d'examiner plus particulièrement le cheminement de ces individus, mentionnons d'abord que la majeure partie d'entre eux proviennent des couches aisées de la petite bourgeoisie, sinon de la bourgeoisie haïtienne.

L'examen des cheminements professionnels en Haïti des femmes intégrées à la petite bourgeoisie au Québec nous montre qu'elles ont une scolarité nettement supérieure à celle des femmes intégrées au prolétariat québécois. Quoique quelques-unes aient émigré pour entreprendre des études universitaires, dans le domaine de la santé particulièrement, d'autres ont reçu une bonne partie de leur formation académique en Haïti. Les principaux champs de formation furent la santé, l'enseignement (niveau secondaire) et le secrétariat. Ce sont surtout les femmes spécialisées en secrétariat qui ont travaillé en Haïti. D'abord formées dans les meilleures écoles, elles ont travaillé soit dans les services de l'administration publique, soit pour des compagnies privées étrangères. Parmi celles qui ont poursuivi des études universitaires, peu ont acquis de l'expérience sur le marché du travail en Haîti, puisque la poursuite des études constituait souvent la raison majeure de leur émigration. 
Les hommes ont en Haïti une scolarité plus élevée que celle des femmes issues de la même classe. Cela s'explique entre autres par le fait que les hommes sont formés essentiellement pour des emplois de type professionnel et technique. On ne voit en effet aucun homme ayant poursuivi des études en secrétariat. Ils se spécialisent plutôt en médecine, en droit, en génie, en architecture, etc. Plusieurs d'entre eux ont également émigré pour pousuivre leur formation universitaire, notamment en Europe et aux Etats-Unis. Si l'éventail de formation de ces hommes est plus grand que celui des femmes, l'expérience de travail acquise en Haïti est également plus variée. En effet, quelques-uns ont travaillé dans le domaine de la santé, du droit, du génie et de l'enseignement supérieur.

L'émigration au Québec de la majeure partie des personnes insérées dans la petite bourgeoisie québécoise s'est donc effectuée dans le sens d'une continuité par rapport à leur formation initiale. De plus, n'ayant aucune obligation familiale en Haĭti, ces personnes ont pu souvent bénéficier, au contraire de celles intégrées au prolétariat québécois, du soutien financier de leur famille.

\section{CONCLUSION}

Dans cet article, nous avons tenté de mettre en évidence le caractère spécifique de l'insertion sur le marché du travail et des trajectoires d'emploi des immigrantes et des jmmigrants haïtiens selon leur destination professionnelle au Québec. Dans ce sens, l'utilisation des données factuelles tirées des récits de vie nous a permis de mieux situer quelques-uns des déterminants expliquant les divers types d'insertion et de trajectoire au Québec.

Les résultats nous confirment d'abord que l'insertion sur le marché du travail et la trajectoire socio-professionnelle diffèrent selon la destination professionnelle au Québec. On constate en effet que 1'insertion des individus dans des emplois de type professionnel et technique au Québec s'effectue dans le sens d'une continuité par rapport à leur cheminement professionnel en Haiti, tandis que leur trajectoire socio-professionnelle présente un profil stable et linéaire. Pour ceux insérés dans des emplois du secteur manufacturier et de celui des services, on constate la précarité de leur situation en termes de statut réglementaire à l'arrivée et de premier emploi occupé, ainsi qu'une trajectoire caractérisée par une rotation d'emploi importante et par de fréquents passages entre secteurs d'activité.

L'insertion sur le marché du travail et les trajectoires socio-professionnelles au Québec varient aussi selon le sexe. Pour les femmes occupant des emplois dans le secteur manufacturier et dans celui des services, on observe une insertion beaucoup plus difficile, puisque le statut réglementaire à l'arrivée (touriste) et le premier emploi occupé (services domestiques) les placent dans une situation encore plus vulnérable. Par ailleurs, même si les femmes intégrées dans des emplois de type professionnel et technique sont plus scolarisées et plus spécialisées, elles n'en sont pas moins limitées à des emplois typiquement féminins, ne se différenciant pas en cela de l'ensemble de la population active féminine. 
Dans un deuxième temps, 1'examen du cheminement professionnel en Haïti, selon 1'appartenance de classe et le sexe des individus, nous amène à constater des différences importantes. Les données nous confirment en effet que l'appartenance de classe en Hä̈ti et le sexe déterminent des cheminements-types en termes de scolarité, de formation et d'expérience de travail. Ainsi, aux couches aisées de la petite bourgeoisie hailtienne sont liées une scolarité moyenne élevée, une formation plus spécialisée et une expérience de travail de type professionnel et technique. Par contre, pour les individus issus du prolétariat haïtien, on remarque un faible niveau de scolarisation et une formation souvent acquise par la pratique même d'un "métier".

Mais l'examen du cheminement professionnel en Haïti des individus issus des couches précaires de la petite bourgeoisie nous montre que les qualifications acquises dans le pays d'origine ne sont pas les seuls facteurs déterminant le type d'insertion et de trajectoire d'emploi au Québec. À cet effet, soulignons le cas d'individus qui, n'ayant pu faire reconnaître leurs qualifications se sont retrouvés dans le secteur manufacturier et dans celui des services. Donc, si l'appartenance de classe en Haïti et le sexe déterminent un processus spécifique d'acquisition des qualifications, la possibilité de les négocier sur le marché du travail au Québec fait appel à d'autres facteurs.

Enfin, si l'enquête ne nous a pas permis d'évaluer précisément l'incidence de la conjoncture économique sur l'insertion et la trajectoire d'emploi des immigrants haïtiens au Québec, elle nous a néanmoins montré quels cheminements ont permis à des personnes de s'insérer dans des emplois de type professionnel et technique durant les années 1960, et à d'autres de s'insérer dans des emplois en manufacture ou dans les services au cours des années 1970 .

\section{RÉFÉRENCES BIBLIOGRAPHIQUES}

BARIL, R., J.-W. GREGORY et R.-L. JACQUES, 1985. "L'exode comme stratégie de survie : le cas du monde rural haítien". A paraître dans Environnement africain.

BERNECHE, Francine, 1983. "Les caractéristiques socio-démographiques de l'immigration caraỉbéenne au Québec au recensement de 1971". In L'immigration caraïbéenne au Canada et au Québec : aspects statistiques. (Sous la direction de V. Piché, S. Larose et M. Labelle). Montréa1, Université de Montréal, Centre de recherches caraïbes, 92-112.

BERNIER, Bernard, 1979. "Main-d'oeuvre féminine et ethnicité dans trois usines de vêtements à Montréall. Anthropologie et Sociétés, 3, 2, 117-139. 
CORTEN, André, 1972. "Migration et intérêts de classe». Nouvelle optique, $8,45-64$.

DEJEAN, Paul, 1978. Les Haïtiens au Québec. Montréal, Presses de I'Université du Québec, 189 p.

HOLLY, D., M. LABELLE et S. LAROSE, 1979. "Dossier: 1'émigration haítienne, un problème national". Collectif Paroles, 2, 18-26.

KEMPENEERS, M. et G. NEILL, 1985. Trajectoires socio-professionnelles des immigrantes et des immigrants haïtiens au Québec. Montréal, Université de Montréa1, Centre de recherches caraïbes, rapport de recherche, $125 \mathrm{p}$.

KEMPENEERS, M. et V. PICHÉ, 1983. "Caractéristiques socio-démographiques de I'immigration caraïbéenne au Québec : 1968-1980". In L'immigration caraïbéenne au Canada et au Québec : aspects statistiques. (Sous la direction de V. Piché, S. Larose et M. Labelle). Montréal, Université de Montréal, Centre de recherches caralbes, 63-71.

LABELLE, M., 1978. Idéologie de couleur et classes sociales en Haïti. Montréal, Presses de l'Université de Montréal, 393 p.

LABELLE, M., S. LAROSE et V. PICHÉ, 1983. "Émigration et immigration : les Haïtiens au Québec". Sociologies et Sociétés, XV, 2, 73-88.

LABELLE, M., D. MEINTEL, G. TURCOTTE et M. KEMPENEERS, 1984. "Immigrées et ouvrières. Un univers de travail à recomposer". Cahiers de recherche sociologique, $2,2,9-47$.

LAROSE, Serge, 1985. Procès migratoire et trajectoire de classe des immigrantes et des immigrants haïtiens au Québec. Montréal, Université de Montréal, Centre de recherches caraíbes, rapport de recherche, 199 p.

NEILL, Ghyslaine, 1983. Trajectoires socio-professionnelles des immigrantes et des immigrants hailtiens au Québec. Montréal, Université de Montréal, Département de démographie, mémoire de maîtrise, $145 \mathrm{p}$.

NORMANDEAU, L. et V. PICHÉ, 1983. "L'emploi projeté des travailleurs et des travailleuses carä̈béens-nes au Québec, 1968-1977". In L'immigration caraïbéenne au Canada et au Québec : aspects statistiques. (Sous la direction de V. Piché, S. Larose et M. Labelle). Montréal, Université de Montréal, Centre de recherches caraïbes, 74-91.

TARDIEU, Camille, 1979. L'immigration haïtienne au Québec de 1968 à 1977. Montréal, Université de Montréal, Département de démographie, mémoire de maîtrise, $140 \mathrm{p}$. 


$$
\text { RÉSUMÉ - SUMMARY - RESUMEN }
$$

NEILL Ghyslaine - CLASSE, SEXE ET TRAJECTOIRE SOCIO-PROFESSIONNELLE : LE CAS DE L'IMMIGRATION HAITIENNE AU QUÉBEC

Si plusieurs travaux ont mis en lumière le rôle de l'immigration haïtienne dans la satisfaction des besoins en main-d'oeuvre du Québec, peu de recherches empiriques ont exploré les mécanismes par lesquels les immigrantes et les immigrants sont amenés à jouer ce rôle. C'est le sexe et l'appartenance de classe dans le pays d'origine qui conditionnent largement les trajectoires socio-professionnelles des immigrants haïtiens au Québec. Ces deux facteurs déterminent en effet un processus spécifique d'acquisition des qualifications, à partir desquelles peuvent se constituer des instruments plus ou moins négociables sur le marché du travail au Québec.

NEILL Ghyslaine - CLASS, SEX AND SOCIO-PROFESSIONAL TRAJECTORY : THE CASE OF HAITIAN IMMIGRATION IN QUEBEC

Various studies have shown the role of Haitian immigration in satisfying the need for manpower in Quebec. Very few, however, analyse the process which leads immigrants to this role. This paper shows that sex and class in the country of origin strongly determine the socio-professional trajectory of Haitian immigrants in Quebec. These two factors define indeed a specific process of skill acquisition, and, therefore, a more or less strong bargaining power on the labor market.

NEILL Ghyslaine - CLASE, SEXO Y TRAYECTORIA SOCIO-PROFESIONAL : EL CASO DE LA INMIGRACIÓN HAITIANA

Si muchos trabajos han puesto a luz el rol de la inmigración haitiana para poder satisfacer las necesidades de mano de obra en Québec, poca busqueda empiricas han explorado los mecanismos por los cuales los y las inmigrantes se ven llevados a jugar este rol. Es el sexo y la pertenancia a una clase en el pais de origen que condicionan ampliamente las trayectorias socio-profesionales de los inmigrantes haitianos en Québec. Estos dos factores determinan pues un proceso específico de adquisición de calificaciones a partir de las cuales pueden constituirse instrumentos más o menos negociables en el mercado de trabajo en Québec. 